\title{
POINTWISE KERNELS OF SCHWARTZ DISTRIBUTIONS
}

\author{
TODOR TODOROV
}

(Communicated by James E. West)

\begin{abstract}
We show that Schwartz distributions have kernels in the class of the pointwise nonstandard functions.
\end{abstract}

The main purpose of this note is to show that every Schwartz distribution $T \in \mathscr{D}^{\prime}$ has a kernel $f:{ }^{*} \mathbb{R}^{d} \rightarrow{ }^{*} \mathbb{C}$ in the class of the pointwise nonstandard functions in the sense that

$$
\langle T, \varphi\rangle=\int_{* \mathbb{R}^{d}} f(x)^{*} \varphi(x) d x
$$

for all $\varphi \in \mathscr{D}$, where ${ }^{*} \varphi$ is the nonstandard extension of $\varphi$. Recall that, in general, the Schwartz distributions do not have kernels in the class of the standard pointwise functions (Schwartz [2]).

We denote the usual classes of the $C^{\infty}$-functions, $C^{\infty}$-functions with compact supports, and continuous complex-valued functions defined on $\mathbb{R}^{d}(d$ is a natural number) by $\mathscr{E} \equiv \mathscr{E}\left(\mathbb{R}^{d}\right) \equiv C^{\infty}\left(\mathbb{R}^{d}\right), \mathscr{D} \equiv \mathscr{D}\left(\mathbb{R}^{d}\right) \equiv C_{0}^{\infty}\left(\mathbb{R}^{d}\right)$, and $C^{0} \equiv C^{0}\left(\mathbb{R}^{d}\right) \equiv C\left(\mathbb{R}^{d}\right)$, respectively, and the class of Schwartz distributions by $\mathscr{D}^{\prime} \equiv \mathscr{D}^{\prime}\left(\mathbb{R}^{d}\right)$. Let $\mathscr{P}$ be the ring of standard complex-valued polynomials defined on $\mathbb{R}^{d}$. As usual, $\mathbb{N}, \mathbb{R}$, and $\mathbb{C}$ will be the systems of the natural, real, and complex numbers, respectively, and we use also the notations $\mathbb{N}_{0}=\{0\} \cup \mathbb{N}$ and $\check{f}(x) \equiv f(-x)$.

In what follows, we shall work in a nonstandard model with a set of individuals $J$ that contains the complex numbers $\mathbb{C}$ and degree of saturation $k$ larger than $2^{\kappa}$ for $\kappa=$ card $C^{0}$. In particular, any polysaturated model of $\mathbb{C}$ will suffice (Stroyan and Luxemburg [3]). If $X$ is a set of complex numbers or a set of (standard) functions, then ${ }^{*} X$ will be its nonstandard extension, and if $f: X \rightarrow Y$ is a (standard) mapping, then ${ }^{*} f:{ }^{*} X \rightarrow{ }^{*} Y$ will be its nonstandard extension. We shall use the same notation, $*$, for the convolution operator $*: \mathscr{D}^{\prime} \times \mathscr{D} \rightarrow \mathscr{E}$ and for its nonstandard extension $*:{ }^{*} \mathscr{D}^{\prime} \times{ }^{*} \mathscr{D} \rightarrow{ }^{*} \mathscr{E}$.

Lemma. There exists $\Delta$ in ${ }^{*} \mathscr{D}$ such that for all $\varphi$ in $C^{0}$ we have

$$
\int_{\bullet_{\mathbb{R}^{d}}} \Delta(x)^{*} \varphi(x) d x=\int_{\cdot_{\mathbb{R}^{d}}} \check{\Delta}(x)^{*} \varphi(x) d x=\varphi(0) .
$$

Received by the editors September 7, 1990.

1980 Mathematics Subject Classification (1985 Revision). Primary 03H05, 30G10, 46F05, 46F10, 46F99.

Key words and phrases. Nonstandard extension, transfer principle, Schwartz distribution, kernel, multiplication of distributions. 
For the proof we refer the reader to [4].

Proposition. If $T$ is a Schwartz distribution, then (1) holds for $f={ }^{*} T * \Delta$ and all $\varphi$ in $\mathscr{D}$.

Proof. Using the properties of the convolution operator, the transfer principle, and the above lemma (since $T * \check{\varphi}$ is in $\mathscr{E}$ ), we obtain

$$
\begin{aligned}
\int_{* \mathbb{R}^{d}}\left({ }^{*} T * \Delta\right)(x)^{*} \varphi(x) d x & =\left\langle{ }^{*} T * \Delta,{ }^{*} \varphi\right\rangle=\left\langle{ }^{*} T,{ }^{*} \varphi * \check{\Delta}\right\rangle \\
& =\left\langle{ }^{*} T *\left({ }^{*} \check{\varphi}\right), \check{\Delta}\right\rangle=\int_{* \mathbb{R}^{d}} \check{\Delta}(x)^{*}(T * \check{\varphi})(x) d x \\
& =(T * \check{\varphi})(0)=\langle T, \varphi\rangle
\end{aligned}
$$

as required.

We shall keep $\Delta$ fixed in what follows.

Corollary. (i) The mapping from $\mathscr{D}^{\prime}$ into ${ }^{*} \mathscr{E}$ defined by $T \rightarrow{ }^{*} T * \Delta$ is injective and preserves the addition, multiplication by a complex (standard) number, and partial differentiation in $\mathscr{D}^{\prime}$.

(ii) There exists an infinitely large natural number $\nu \in{ }^{*} \mathbb{N}$ such that $P * \Delta=P$ holds for all (nonstandard, in general) polynomials $P \in{ }^{*} \mathscr{P}$ with degree not higher than $\nu$. In particular, ${ }^{*} P * \Delta={ }^{*} P$ holds for all standard polynomials $P \in \mathscr{P}$.

(iii) If $f$ is a continuous function, then ${ }^{*} f * \Delta$ is an extension of $f$.

Proof. (i) By the transfer principle, ${ }^{*} T * \Delta \in{ }^{*} \mathscr{E}$ for all $T$ in $\mathscr{D}^{\prime}$, while ${ }^{*} T * \Delta=0$ (in ${ }^{*} \mathscr{E}$ ) implies $T=0$ (in $\mathscr{D}^{\prime}$ ), by the above proposition. The preservation of linear operations follows immediately from the corresponding property of the convolution operator and transfer principle.

(ii) Define the internal set

$$
\Omega_{\Delta}=\left\{n \in{ }^{*} \mathbb{N}: \int_{* \mathbb{R}^{d}} \Delta(x) x^{\alpha} d x=0, \quad 1 \leq|\alpha| \leq n, \alpha \in{ }^{*} \mathbb{N}_{0}^{d}\right\}
$$

and observe that, by our lemma, $\mathbb{N} \subseteq \Omega_{\Delta}$. Hence, by overflow, $\Omega_{\Delta}$ contains an infinitely large number $\nu$. Suppose now, that $\xi \in{ }^{*} \mathbb{R}^{d}$. The hyperfinite (*-finite) Taylor's expansion of $P$ at $\xi$ gives

$$
\begin{aligned}
(\Delta * P)(\xi) & \equiv \int_{\mathbb{R}^{d}} \Delta(x) P(\xi-x) d x \\
& =P(\xi)+\sum_{|\alpha|=1}^{\nu} \frac{(-1)^{|\alpha|} \partial^{\alpha} P(\xi)}{\alpha !} \int_{* \mathbb{R}^{d}} \Delta(x) x^{\alpha} d x=P(\xi),
\end{aligned}
$$

as required. The equality ${ }^{*} P * \Delta={ }^{*} P$ follows as a particular case since the degree of a standard polynomial is always finite and hence less than $\nu$.

(iii) follows immediately from our lemma for $\varphi=f_{x}$ and standard $x \in \mathbb{R}^{d}$, where $f_{x}(\xi)=f(x-\xi)$. The proof is complete.

Remark (Multiplication of distributions). Consider $* \mathscr{E}$ as a differential algebra over ${ }^{*} \mathbb{C}$ with respect to pointwise addition, multiplication, and internal partial differentiation. Notice now that the space of Schwartz distributions $\mathscr{D}^{\prime}$ is isomorphically embedded in $* \mathscr{E}$ through the above injection and hence the 
Schwartz distributions can be multiplied within an associative algebra (something impossible in $\mathscr{D}^{\prime}$ itself). Further, the operations in ${ }^{*} \mathscr{E}$ generalize the usual operations with polynomials in the sense the $\mathscr{P}$ (considered as a subset of $\mathscr{D}^{\prime}$ ) is a differential subalgebra of $* \mathscr{E}$ over $\mathbb{C}$. The multiplication in $* \mathscr{E}$ also generalizes the usual multiplication in $C^{0}$ (considered as a subset of $\mathscr{D}^{\prime}$ ) although in a somewhat weaker sense: if $f$ and $g$ are two continuous functions and ${ }^{*} f * \Delta$ and ${ }^{*} g * \Delta$ are their images in $* \mathscr{E}$, then their product $\left({ }^{*} f * \Delta\right)\left({ }^{*} g * \Delta\right)$ in $* \mathscr{E}$ is an extension of the usual product $f g$ in $C^{0}$. We wish to pay attention to the similarity between the class of nonstandard functions $* \mathscr{E}$ (in the context discussed above) and the classes of generalized functions introduced (in the framework of standard analysis) by Colombeau [1] with the same purpose: multiplication of Schwartz distributions.

\section{REFERENCES}

1. J. F. Colombeau, New generalized functions and multiplication of distributions, NorthHolland Math. Stud., vol. 84, North-Holland, Amsterdam and New York, 1984.

2. L. Schwartz, Theorie des distributions I, II, Herman, Paris, 1950, 1951.

3. K. D. Stroyan and W. A. J. Luxemburg, Introduction to the theory of infinitesimals, Academic Press, New York, 1976.

4. T. Todorov, A nonstandard delta function, Proc. Amer. Math. Soc. 110 (1990), 1143-1144.

Department of Mathematics, California Polytechnic State University, San luis OBispo, CALIFORNIA 93407 